\title{
CÁLCULO DE LA INCERTIDUMBRE EN LA DETERMINACIÓN DE PLAGUICIDAS ORGANOCLORADOS Y TRIAZOLES EN CAFÉ VERDE POR GC-MS
}

\section{UNCERTAINTY CALCULATION IN THE QUANTITATION OF ORGANOCLORINE AND TRIAZOLE PESTICIDES IN GREEN COFFEE BY GC-MS}

\author{
Hugo Fernando ARIAS', Juan Pablo ARRUBLA', Ana Isabel GIRALDO1 \\ ${ }^{1}$ Universidad Tecnológica de Pereira, Facultad de Tecnología, Escuela de Tecnología Química, \\ Grupo de Investigación de Oleoquímica, Carrera 27 \# 10-02, Pereira, Colombia. \\ *Autor corresponsal.E-mail: juanpablo77@utp.edu.co
}

Historia del artículo

Recibido: Noviembre 24, 2017

Evaluado: Marzo 13, 2018

Aceptado: Abril 18, 2018

Disponible: Mayo 23, 2018

\section{Resumen}

En el presente trabajo se describe la implementación de una metodología multirresiduo para la determinación de plaguicidas organoclorados y triazoles en café verde. Los plaguicidas se extrajeron con una mezcla de acetronitrilo-agua (5:2), seguida de una partición líquido-líquido y una extracción en fase sólida con fase reversa RP18. Los extractos se limpiaron posteriormente por extracción en fase sólida con cartuchos de carbón activado-NH2 para eliminar interferencias. La determinación analítica se realizó por cromatografía de gases con inyección splitless acoplada a espectrometría de masas. Se obtuvieron porcentajes de recuperación por encima del 70\%. Se evaluó la precisión instrumental y la linealidad en un rango de concentraciones inferior a los límites máximos de residualidad de cada plaguicida. Finalmente, se estimó la incertidumbre de las mediciones y se obtuvo que todas las incertidumbres estuvieron por debajo del $15 \%$, al igual que los porcentajes de error en las concentraciones medidas.

Palabras clave: café; cromatografía de gases; espectrometría de masas; incertidumbre; plaguicidas

Abstract |

Implementation of a multiresidue methodology for the determination of organochlorine and triazole pesticides in green coffee is described. Pesticides were extracted with a mixture of acetonitrile-water (5:2), followed by liquid -liquid partition and solid-phase extraction with reversed phase RP18. Extracts were subsequently cleaned by solid phase extraction with activated carbon- $\mathrm{NH}_{2}$ cartridges to eliminate interferences. Analytical determination was made by gas chromatography coupled to mass spectrometry with splitless injection. Recovery percentages were all above $70 \%$. Instrumental precision and linearity were evaluated below the maximum residual limits of each pesticide. Finally, uncertainty and inaccuracy were below $15 \%$ for all our measurements.

Keywords: coffee; gas chromatography; mass spectrometry; uncertainty; pesticides

\section{INTRODUCCIÓN |}

El café se ha constituido, en el marco de la economía colombiana, en uno de los productos más importantes de la agricultura. Colombia se ha posicionado como productor de unos de los cafés suaves más apetecidos del mundo y es el principal producto agrícola de exportación, ya que representa el 52\% de las exportaciones de todo el sector agropecuario (Espinal et al., 2006), con una cosecha cercana a 12.9 millones de sacos anuales (Federación Nacional de Cafeteros, 2017); sus exportaciones se destinan a Estados Unidos, Japón y Bélgica, con el $41.3 \%$, el $9.3 \%$ y el $8.2 \%$, respectivamente (Dinero, 2015). 
El componente agrícola de la cadena productiva de café (siembra, cosecha, recoleccióny secado)es altamentegenerador de empleo, con una ocupación aproximada de 500.000 empleos directos. Los ingresos llegan a más de dos millones de personas en la zona rural, por lo que se considera el principal dinamizador de la economía rural en Colombia (Muñoz, 2012).

En el cultivo, el almacenamiento y el trasporte del café se usan pesticidas, como, por ejemplo, insecticidas, herbicidas, nematicidas y fungicidas que, de no aplicarse de manera controlada, podrían aportar residuos al producto final; además, algunas especies de plantas han demostrado que tienen mayores factores de bioacumulación. Esta acumulación de pesticidas en la planta puede tener graves consecuencias a través de la cadena alimentaria, lo que representa un potencial de efectos nocivos para los seres humanos (Juraske et al., 2009; Landau-Ossondo et al., 2009).

El uso de pesticidas en la agricultura es, por tanto, objeto de un seguimiento permanente debido al riesgo potencial que suponen para la salud pública y los ecosistemas. Por esta razón, se han establecido límites máximos de residualidad permitidos de acuerdo con parámetros nacionales e internacionales (Dallos y Guerrero, 2005).

Internacionalmente, es la FAO (Food and Agriculture Organization of United Nations) la que establece los límites máximos de residuos de pesticidas (LMR) para alimentos (World Health Organization y Food and Agriculture Organization of United Nations, 2017). A nivel nacional, el Ministerio de Agricultura y Desarrollo Rural y el Ministerio de la Protección Social promulgaron, en el 2007, la Resolución 2906, por la cual se establecen los LMR en alimentos para consumo humano, entre los cuales se encuentra el café en grano (Instituto Colombiano Agropecuario, 2017; Ministerio de Agricultura y Desarrollo Rural y de la Protección Social, 2007).

En el análisis de la residualidad se hace necesario el apoyo de laboratorios acreditados que cuenten con métodos de análisis confiables, sensibles y robustos. La cromatografía de gases acoplada a la espectrometría de masas es una técnica con la capacidad de cuantificar pesticidas a las concentraciones exigidas por la legislación vigente, y sirve de apoyo para los organismos de control en la certificación de productos agrícolas de exportación.

Una de las inquietudes en la medición de los niveles de residualidad se encuentra en la imperfección natural de la realización de las mediciones, lo que hace imposible conocer con certeza absoluta el valor verdadero del mensurando. Toda medición lleva implícita una incertidumbre. La incertidumbre, junto con la trazabilidad, es uno de los conceptos metrológicos fundamentales; es el parámetro asociado con el resultado de la medición que caracteriza la dispersión de los valores. Este parámetro, por lo general, se asocia con desviación estándar, lo que indica un cierto intervalo de confianza o de distribución más probable de los valores repetitivos de una medición, pero la diferencia determinante es el hecho de que el concepto de in- certidumbre está íntimamente ligado con el concepto de trazabilidad, no así el de precisión (Órgano Nacional de Acreditación de la República de Cuba, 2016; Schmid et al., 2004). El término incertidumbre pretende ser más globalizador en el sentido de considerar todas las fuentes de error que intervienen en el resultado final y porque permite identificar los puntos clave del proceso de medida, así como las etapas susceptibles de mejora.

Existen diversas normas internacionales que sirven de guía en el cálculo de la incertidumbre. En específico, para los ensayos químicos se pueden mencionar las guías desarrolladas por Lupac, Eurachem, Citac y Nist. En ellas se plantean metodologías específicas para estimar la incertidumbre en este tipo de mediciones (Órgano Nacional de Acreditación de la República de Cuba, 2016).

El presente artículo describe la implementación de una metodología multirresiduo de cromatografía de gases acoplada a espectrometría de masas para la determinación de pesticidas organoclorados y triazoles en café verde de origen colombiano destinado a exportación, con el fin de certificar la inocuidad del producto. Asimismo, se describe el cálculo de la incertidumbre de la metodología al involucrar todas las etapas de la determinación.

\section{MATERIALES Y MÉTODOS}

\section{Materiales de referencia, reactivos y soluciones}

Los plaguicidas de referencia con porcentajes de pureza entre eL $97,5 \%$ y el $99,5 \%$ se obtuvieron de ChemService (West Chester, EE.UU.). La acetona, el hexano, el tolueno, el metanol y el acetonitrilo se obtuvieron de J. T. Beaker y Merck. A partir de cada estándar se prepararon soluciones patrón a una concentración de $5000 \mu \mathrm{g} / \mathrm{mL}$; en la (Tabla 1) se relacionan los solventes que se utilizaron para cada plaguicida.

Se preparó una mezcla de los plaguicidas con base en su límite máximo de residualidad (LMR). Para esto se agregaron los volúmenes necesarios con el fin de que cada componente quedará en una concentración igual a 200 veces el LMR en acetona-hexano (1:1); en el caso del disulfotón, la concentración equivale a 100 veces el LMR. A partir de esta mezcla se realizaron las diluciones para los niveles de calibración en acetona-hexano (1:1), las cuales se prepararon en concentraciones entre $0.005 \mu \mathrm{g} / \mathrm{mL}$ y $0.8 \mu \mathrm{g} / \mathrm{mL}$. Para los ensayos de extracción se utilizaron cartuchos en fase sólida RP18 (100 mg, $6 \mathrm{~mL}$ ), y para la limpieza de los extractos cartuchos en fase sólida Envicarb- $\mathrm{NH}_{2}(500 \mathrm{mg} / 500 \mathrm{mg}, 6 \mathrm{~mL})$.

\section{Obtención y preparación de la muestra}

Se analizaron muestras de café verde en grano calidad excelso tipo exportación suministradas por la Federación Nacional de Cafeteros, y se conservaron en bolsas depolietileno a temperatura ambiente. Se procesaron aproximadamente 15 gramos de café verde, y se molieron en un molino marca IKA referencia MF 10 Basic a 5500 rpm. Posteriormente, se pesaron y sometieron al proceso de extracción. 
Tabla 1. Disolventes para la preparación de disoluciones patrón de plaguicidas individuales y el límite máximo de residualidadd (LMR).

\begin{tabular}{cccc}
\hline ITEM & NOMBRE & SOLVENTE & LMR $(\mathrm{mg} / \mathrm{kg})$ \\
\hline 1 & Forato & Tolueno & 0.02 \\
2 & Terbufós & Metanol & 0.05 \\
3 & Disulfotón & Metanol & 0.2 \\
4 & Clorpirifós & Acetona-hexano (1:1) & 0.05 \\
5 & Triadimefón & Acetona-hexano (1:1) & 0.05 \\
6 & Triadimenol & Tolueno-acetonitrilo (1:1) & 0.1 \\
7 & Propiconazol I \& II & Acetona-hexano (1:1) & 0.1 \\
\hline
\end{tabular}

\section{Instrumentación}

Las mediciones se realizaron en un cromatógrafo de gases acoplado a espectrometría de masas marca Shimadzu, referencia QP 2010, equipado con inyector automático referencia AOC 20i+s. Para el análisis y el procesamiento de los datos se empleó el software GCMs lab-solution versión 2.5 .

\section{Condiciones cromatográficas}

Se utilizó una columna capilar marca Restek, referencia RTX 5Sil MS (30 m x 0.25 mm I.D.; $0.25 \mu \mathrm{m} \mathrm{fd).} \mathrm{Las} \mathrm{condiciones}$ cromatográficas utilizadas en el análisis de los plaguicidas fueron las siguientes: volumen de inyección $2 \mu \mathrm{L}$; inyección en modo splitless, flujo de purga de $3 \mathrm{~mL} / \mathrm{min}$ y temperatura del inyector de $250^{\circ} \mathrm{C}$. El gas de transporte fue helio con un flujo de $1 \mathrm{~mL} / \mathrm{min}$. El programa de temperaturas del horno se muestra en la (Tabla 2), y las condiciones de detección en la (Tabla 3).

\section{Procedimiento de extracción}

Se tomaron 10 gramos de café verde molido y se sometieron a extracción por ultrasonido con $30 \mathrm{~mL}$ de una solución de acetonitrilo-agua (5:2) durante 15 minutos, luego se filtró. Al residuo sólido se adicionan $20 \mathrm{~mL}$ de acetonitrilo y se agitaron durante 15 minutos en el agitador orbital, luego se filtró. Se combinaron los filtrados y se realizó una extracción líquido-líquido con $30 \mathrm{~mL}$ de solución salina en buffer fosfato $\mathrm{pH} 7$. Se tomó la fase orgánica y se pasó a través de un cartucho para extracción en fase sólida RP18 (1000 mg, $6 \mathrm{~mL}$ ), previamente acondicionado con $10 \mathrm{~mL}$ de acetonitrilo. El efluente se recogió en un matraz volumétrico (clase A) de $50 \mathrm{~mL}$, luego se adicionaron al matraz $12.5 \mathrm{~mL}$ de tolueno y se aforó con acetonitrilo. Se vertió la mezcla resultante a un erlenmeyer de $100 \mathrm{~mL}$ y se secó con $5 \mathrm{~g}$ sulfato de sodio anhidro (Japan Mistry of Health, Labour and Welfare, 2006; Guo, Lv, Tan y Yu, 2009; Pizzutti et al., 2012; Yang et al., 2011).

\section{Procedimiento de limpieza}

Para el proceso de limpieza se empleó un cartucho de Envicarb- $\mathrm{NH}_{2}$, el cual antes se acondicionó con $10 \mathrm{~mL}$ de acetonitrilo-tolueno (3:1). La muestra se pasó a través del cartucho y el efluente se llevó a rotaevaporación. Se adicionaron $10 \mathrm{~mL}$ de acetona y se evaporó de nuevo el solvente; luego se adicionaron $5 \mathrm{~mL}$ más de acetona y se llevó hasta sequedad. Finalmente, se recogió el extracto seco con $1.5 \mathrm{~mL}$ de una mezcla de acetona-hexano (1:1), y se transfirió a un vial de inyección ajustando el volumen a $1 \mathrm{~mL}$ (Japan Mistry of Health, Labour and Welfare, 2006; Guo et al., 2009; Pizzutti et al., 2012; Yang et al., 2011).

Tabla 2. Condiciones cromatográficas para la separación.

\begin{tabular}{cc}
\hline VARIABLE & VALOR \\
\hline Temperatura 1 & $120^{\circ} \mathrm{C}$ \\
Tiempo 1 & Dos minutos \\
Rampa 1 & $10^{\circ} \mathrm{C} /$ min \\
Temperatura 2 & $270^{\circ} \mathrm{C}$ \\
Tiempo 2 & 0 minutos \\
Rampa 2 & $25^{\circ} \mathrm{C} /$ min \\
Temperatura final & $320^{\circ} \mathrm{C}$ \\
Tiempo final & Dos minutos
\end{tabular}

Tabla 3. Condiciones de detección del espectrómetro de masas.

\begin{tabular}{cc}
\hline VARIABLE & VALOR \\
Temperatura de interface & $280^{\circ} \mathrm{C}$ \\
Temperatura fuente de iones & $260^{\circ} \mathrm{C}$ \\
Modo de ionización & Impacto electrónico $(70 \mathrm{eV})$ \\
Modo de lectura & $\mathrm{SIM}$ \\
\hline
\end{tabular}

\section{RESULTADOS Y DISCUSIÓN}

\section{Análisis cualitativo}

A fin de verificar la identidad de los plaguicidas y asignar los nombres a los picos cromatográficos correspondientes, se tomaron sus espectros de masas en full scan y se compararon con los de la base de datos WileyAccessPak 7th Edition. John Wiley \& Sons Inc., y se verificó que los índices de similitud fueran superiores al 80\%. En la (Tabla 4) se presentan los índices de similitud encontrados para cada plaguicida.

Tabla 4. Similitud de los espectros experimentales con la base de datos.

\begin{tabular}{cc}
\hline PLAGUICIDA & ÍNDICE DE SIMILITUD \\
\hline Forato & $94 \%$ \\
Terbufós & $95 \%$ \\
Disulfotón & $96 \%$ \\
Clorpirifós & $90 \%$ \\
Triadimefón & $94 \%$ \\
Triadimenol & $93 \%$ \\
Propiconazol I \& II & $81 \%$ \\
\hline
\end{tabular}




\section{Curvas de calibración, linealidad y precisión instrumental}

La relación entre las concentraciones del nivel 1 y 6 fue de 1 a 32. Una inspección visual de la gráfica de calibrado permitió observar la relación lineal entre la señal y la concentración. En la (Tabla 5) se registran los rangos de concentración y los parámetros de regresión.

Cada concentración se inyectó por triplicado y se evaluó la precisión instrumental en los diferentes puntos de las curvas de calibración con base en la desviación estándar de las áreas bajo cada pico correspondiente. En general, las desviaciones estándar relativas (\%DER) se mantuvieron por debajo del $5 \%$, con la excepción de forato y propiconazol, para los cuales en el primer nivel fue del 5,94\% y 6,49\%, respectivamente. Sin embargo, si se tiene en cuenta que la variación en los extremos de la curva es mayor que en el centro de gravedad de esta (Schmid et al., 2004), cabría esperar mayor variabilidad en los primeros niveles; además, porque los datos se acercan al ruido instrumental. En conclusión, los valores de \%DER para forato y propiconazol en el primer nivel son aceptables.

\section{Límites de detección (LOD) y cuantificación (LOQ)}

Se calculó la relación señal ruido (S/R) de todos los picos cromatográficos en cada uno de los niveles de calibración. De cada plaguicida se obtuvieron tres datos de relación señal ruido por cada concentración. Con los datos de concentración y relación señal ruido promedio se realizó una regresión lineal, y con la ecuación resultante se calculó la concentración necesaria para obtener las relaciones señal ruido de 3 y 10, correspondientes al límite de detección y cuantificación, respectivamente. En la (Tabla 6) se presentan los resultados para todos los plaguicidas.

Todos los límites de cuantificación calculados estuvieron por debajo de la concentración menor en su respectiva curva de calibración, excepto clorpirifós y triadimefón. Sin embargo, la precisión del instrumento en los niveles menores de estos dos plaguicidas es inferior al 5\%, lo que significa que se puede cuantificar confiablemente en estas concentraciones a pesar de que el límite de cuantificación calculado sea mayor.

Tabla 5. Rangos de concentración y parámetros de regresión para cada curva.

\begin{tabular}{|c|c|c|c|c|c|}
\hline \multicolumn{6}{|c|}{ RESULTADOS DE REGRESIÓN } \\
\hline PLAGUICIDA & RANGO DE CONCENTRACIÓN (MG/L) & PENDIENTE & INTERCEPTO & COEFICIENTE DE CORRELACIÓN & $\mathbf{R}^{2}$ \\
\hline Forato & $0.0050-0.1600$ & 2179914 & -3692 & 0.998 & 0.996 \\
\hline Disulfotón & $0.0250-0.8000$ & 1684304 & -13602 & 0.999 & 0.998 \\
\hline Clorpirifós & $0.0125-0.4000$ & 167325 & -1921 & 0.999 & 0.998 \\
\hline Triadimenol & $0.0250-0.8000$ & 305146 & -9086 & 0.997 & 0.994 \\
\hline $\begin{array}{c}\text { Propiconazol } \\
\text { I \& II }\end{array}$ & $0.0250-0.8000$ & 542550 & -8425 & 0.998 & 0.997 \\
\hline
\end{tabular}

Tabla 6. Límites de detección y de cuantificación de los plaguicidas.

\begin{tabular}{ccc}
\hline PLAGUICIDA & LíMITE DE DETECCIÓN (MG/L) & LíMITE DE CUANTIFICACIÓN (MG/L) \\
\hline Forato & 0.0048 & 0.0051 \\
Terbufós & 0.0078 & 0.0080 \\
Disulfotón & 0.015 & 0.015 \\
Clorpirifós & 0.016 & 0.017 \\
Triadimefón & 0.013 & 0.015 \\
Triadimenol & 0.021 & 0.021 \\
Propiconazol I \& II & 0.014 & 0.015
\end{tabular}




\section{Efecto matriz}

El análisis del efecto matriz se determinó mediante la relación señal-ruido (S/R) (Aguirre, Pérez y Pujol, 2001), debido a que los patrones con los que se construyó la curva de calibración fueron soluciones en las que solo estuvieron presentes los analitos y el solvente; por tanto, la relación señal ruido calculada sobre estos patrones no refleja el efecto de la matriz sobre la señal de fondo del instrumento. Si bien el procesamiento de la muestra busca, además de extraer los analitos, retirar la mayor cantidad posible de constituyentes de matriz, algo de estos constituyentes logra llegar al extracto final. Por esta razón fue necesario evaluar el efecto que estos interferentes tienen sobre el ruido y las consecuencias sobre los límites de detección y cuantificación. Para esto se procesaron 15 muestras de café verde libre de plaguicidas y se calculó la relación señal ruido en los intervalos de tiempo en que aparecen los picos de los plaguicidas. El promedio de estos datos se multiplicó por tres y diez para recalcular los límites de detección y cuantificación. Los resultados se muestran en las (tablas 7 y 8 ).

Tabla 7. Relación señal ruido de las muestras de café verde en blanco para evaluar el efecto de la matriz.

\begin{tabular}{|c|c|c|c|c|c|c|c|}
\hline \multirow{2}{*}{ RÉPLICA } & FORATO & TERBUFÓS & DISULFOTÓN & CLORPIRIFÓS & TRIADIMEFÓN & TRIADIMENOL & PROPICONAZOL \\
\hline & $\mathbf{S} / \mathbf{R}$ & $\mathbf{S} / \mathbf{R}$ & $\mathbf{S} / \mathbf{R}$ & $S / \mathbf{R}$ & $\mathbf{S} / \mathbf{R}$ & $\mathbf{S} / \mathbf{R}$ & $\mathbf{S} / \mathbf{R}$ \\
\hline 1 & 2.47 & 18.84 & 0.29 & 5.47 & 4.52 & 1.10 & 0.77 \\
\hline 2 & 2.11 & 20.39 & 0.15 & 16.12 & 3.12 & 0.03 & 0.64 \\
\hline 3 & 2.69 & 11.71 & 0.47 & 8.64 & 2.95 & 1.48 & 2.43 \\
\hline 4 & 2.51 & 24.07 & 0.37 & 11.30 & 6.10 & 1.56 & 1.24 \\
\hline 5 & 0.98 & 18.84 & 2.81 & 15.09 & 1.14 & 0.16 & 2.01 \\
\hline 6 & 1.53 & 4.61 & 1.11 & 2.68 & 2.39 & 2.08 & 0.43 \\
\hline 7 & 1.33 & 13.65 & 1.28 & 24.14 & 5.08 & 0.92 & 0.98 \\
\hline 8 & 1.39 & 7.91 & 0.96 & 13.79 & 4.24 & 1.50 & 0.79 \\
\hline 9 & 0.87 & 17.14 & 1.91 & 17.68 & 1.69 & 1.13 & 1.68 \\
\hline 10 & 2.70 & 26.43 & 0.12 & 5.67 & 0.97 & 3.67 & 0.56 \\
\hline 11 & 1.70 & 21.56 & 0.21 & $19 \cdot 39$ & 1.65 & 1.31 & 2.46 \\
\hline 12 & 1.21 & 11.45 & 2.01 & 11.76 & 4.95 & 0.42 & 1.55 \\
\hline 13 & 2.57 & 14.87 & 1.57 & 21.40 & 2.79 & 2.63 & 1.96 \\
\hline 14 & 1.47 & 9.89 & 1.68 & $9 \cdot 32$ & 0.52 & 0.57 & 0.59 \\
\hline 15 & 0.79 & 10.54 & 3.51 & 14.87 & 2.48 & 1.08 & 1.47 \\
\hline Promedio & 1.75 & 15.46 & 1.23 & 13.15 & 2.97 & 1.31 & 1.30 \\
\hline$(\mathrm{s} / \mathrm{R})^{*} 3$ & 5.26 & 46.38 & 3.69 & 39.46 & 8.92 & 3.93 & 3.91 \\
\hline$(S / R) * 10$ & 17.55 & 154.60 & 12.30 & 131.54 & 29.73 & 13.10 & 13.04 \\
\hline
\end{tabular}

Tabla 8. Límites de detección y cuantificación al considerar el efecto matriz.

\begin{tabular}{ccc}
\hline PLAGUICIDA & $\begin{array}{c}\text { LíMITE DE } \\
\text { DETECCIÓN (MG/L) }\end{array}$ & $\begin{array}{c}\text { LíMITE DE CUANTIFI- } \\
\text { CACIÓN (MG/L) }\end{array}$ \\
\hline Forato & 0.0049 & 0.0053 \\
Terbufós & 0.0094 & 0.014 \\
Disulfotón & 0.015 & 0.015 \\
Clorpirifós & 0.019 & 0.025 \\
Triadimefón & 0.015 & 0.022 \\
Triadimenol & 0.021 & 0.021 \\
Propiconazol I \& II & 0.015 & 0.016 \\
& & \\
\hline
\end{tabular}

Se puede observar en la (tabla 7) que el resultado de multiplicar la relación señal ruido por tres nunca supera ninguno de los datos individuales, lo que se encuentra conforme con la definición y, sobre todo, con la justificación de límite de detección. La alta variabilidad en los datos es de esperarse puesto que nos encontramos en el nivel del ruido.

La (tabla 9) presenta la comparación entre el límite de cuantificación calculado a partir de la curva de calibración (LC1), el límite de cuantificación con el efecto de matriz (LC-2) y el valor de concentración mínimo de la curva de calibración (N1).

Se observa en la (tabla 9) que la mayor afectación por matriz la sufrieron terbufós, clorpirifós y triadimefón. Esto no representa un problema puesto que el límite de cuantificación, a pesar de todo, se mantiene 20 veces por debajo del LMR. 
Tabla 9. Comparación de los límites de cuantificación.

\begin{tabular}{cccc}
\hline PESTICIDA & LC-1 $(\mathbf{M G} / \mathrm{L})$ & LC-2 $(\mathbf{M G} / \mathrm{L})$ & N1 $(\mathrm{MG} / \mathrm{L})$ \\
\hline Forato & 0.0051 & 0.0053 & 0.0050 \\
Terbufós & 0.0080 & 0.014 & 0.013 \\
Disulfotón & 0.015 & 0.015 & 0.025 \\
Clorpirifós & 0.017 & 0.025 & 0.013 \\
Triadimefón & 0.015 & 0.022 & 0.013 \\
Triadimenol & 0.021 & 0.021 & 0.025 \\
Propiconazol I \& II & 0.015 & 0.016 & 0.025 \\
\hline
\end{tabular}

LC-1 es el límite de cuantificación calculado de la gráfica de calibración sin tener en cuenta el efecto de la matriz en la señal. LC-2 es el límite de cuantificación al considerar el efecto de la matriz en la señal. N1 es el nivel de menor concentración usado en la curva de calibración.

\section{Porcentajes de recuperación}

A fin de evaluar los porcentajes de recuperación se preparó una solución de referencia con concentraciones igual a cinco veces el LMR de cada plaguicida, se tomaron tres muestras de 10 g de café verde molido libre de plaguicidas y se adicionó a cada una de las muestras $1 \mathrm{~mL}$ de la solución de referencia; luego se sometieron a extracción y se realizó la lectura instrumental de los extractos, se calculó el porcentaje de recuperación como la relación entre el área de cada analito en cada una de las muestras y el área de cada analito en la solución de referencia, con el objetivo de evitar el error por interpolación. En la (tabla 10) se muestran los resultados de los porcentajes de recuperación para cada plaguicida.

Tabla 10. Porcentajes de recuperación en la extracción de plaguicidas a partir de muestras de café verde en grano.

\begin{tabular}{ccccccc}
\hline PLAGUICIDA & $\mathbf{1}$ & $\mathbf{2}$ & $\mathbf{3}$ & PROMEDIO & $\begin{array}{c}\text { DESVIACIÓN } \\
\text { ESTÁNDAR }\end{array}$ & \%DER* \\
\hline Forato & 73.5 & 81.4 & 67.9 & 74.3 & 6.8 & 9.13 \\
Terbufós & 84.3 & 91.8 & 89.9 & 88.7 & 3.9 & 4.43 \\
Disulfotón & 87.6 & 96.0 & 98.0 & 93.9 & 5.5 & 5.88 \\
Clorpirifós & 92.7 & 85.4 & 78.5 & 85.5 & 7.1 & 8.30 \\
Triadimefón & 68.0 & 75.4 & 72.4 & 71.9 & 3.7 & 5.13 \\
Triadimenol & 81.0 & 86.2 & 93.2 & 86.8 & 6.1 & 7.04 \\
Propiconazol & 88.0 & 97.9 & 93.7 & 93.2 & 5.0 & 5.35 \\
I \& II & & & & & & \\
\hline
\end{tabular}

*\%ER: desviación estándar relativa

Todos los porcentajes de recuperación promedio estuvieron entre el $70 \%$ y el $95 \%$, con una baja dispersión de los datos (\%DER por debajo del 10\%). Es importante que las desviaciones estándar relativas (dispersiones) sean pequeñas, ya que influyen directamente en la incertidumbre de medición, es decir, en la precisión del método. Además, proporciona una idea de la reproducibilidad en la extracción.

\section{Incertidumbre}

Para la determinación de la incertidumbre (Ellison, WiIliams y Eurachem Working Group on Uncertainty in Chemical
Measurement, 2012) se inició con la realización de un esquema del proceso, y se observaron así de manera independiente sus etapas. Véase la (Fig. 1).

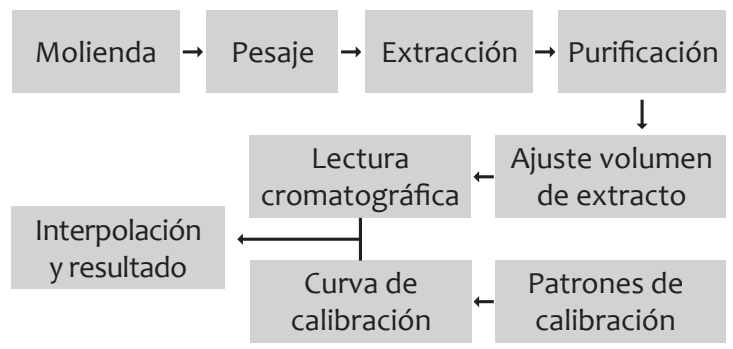

Figura 1. Diagrama del método de determinación de plaguicidas en café verde.

Inicialmente, se debe considerar que las moléculas de plaguicidas están contenidas en un medio sólido que constituye la matriz, en este caso el café. Con el fin de cuantificarlas se extraen con un solvente adecuado para la medición instrumental; el porcentaje de recuperación en la extracción y el volumen final de extracto, así como la masa inicial de matriz, constituyen variables de entrada en el cálculo de la concentración que es la variable de salida. La otra variable de entrada es la concentración de plaguicida en el extracto determinada en la lectura instrumental. El modelo matemático se describe a continuación:

$$
C_{m}=100 \frac{C_{e x t} * V_{e x t}}{\% R * m_{m}} \text { (1) }
$$

Donde:

- $C_{m}=$ concentración de plaguicida en la muestra $(\mathrm{mg} / \mathrm{kg})$.

- $\mathrm{C}_{\text {ext }}=$ concentración de plaguicida en el extracto $(\mathrm{mg} / \mathrm{L})$.

- $\mathrm{V}_{\text {ext }}=$ volumen de extracto $(\mathrm{mL})$.

- $\quad \% \mathrm{R}=$ porcentaje de recuperación (no tiene unidades).

- $\mathrm{m}_{\mathrm{m}}=$ masa de la muestra (g).

Lo anterior permitió establecer como fuentes de incertidumbre:

1. La pesada de la muestra cuya variabilidad se obtuvo del certificado de calibración; la incertidumbre expandida esta expresada en la ecuación (2), posteriormente la muestra se sometió al proceso de extracción y limpieza de los plaguicidas; este proceso conllevó pérdidas de analito que se evaluaron al procesar por triplicado muestras contaminadas con concentraciones conocidas: todas las incertidumbres resultantes de las mediciones de volumen fortificadas en el proceso (es decir, del uso de pipetas, probetas, etc.), quedaron incluidas en el cálculo de incertidumbre por recuperación (Ecuación 3).

$$
U=(0.00010+0.0000013 m) / x
$$

En donde $X$ corresponde al factor de cobertura y $m$ a la masa de la muestra (g). Para el forato se determinó la incertidumbre estándar en la pesada con un factor de cobertura de 2, como se indica a continuación: 
Para la determinación del cálculo de la incertidumbre por recuperación se tiene que $\mathrm{DS}_{\% \mathrm{R}}$ corresponde a la desviación estándar del porcentaje de recuperación promedio y $\mathrm{n}$ al número de mediciones.

$$
U(\% R)=\frac{D S_{\% R}}{\sqrt{n}}(3)
$$

Para el forato se obtuvo una recuperación promedio del $74,3 \%$, con una desviación estándar de 6,8\% para tres repeticiones. Así, entonces:

$$
U(\% R)=\frac{6,80}{\sqrt{3}}=3.93
$$

2. Al final de la extracción y la limpieza, los analitos se transfirieron al vial de inyección y se ajustó el volumen de extracto a $1 \mathrm{ml}$. Estos viales se calibraron por pesada y la incertidumbre en el volumen se calculó a partir de la dispersión de las masas resultantes (Ecuación 4), en donde $\mathrm{DS}_{\mathrm{x}}$ corresponde a la desviación estándar de los valores promedio de la medición por gravimetría y $\mathrm{n}$ al número de mediciones.

$$
U(v o l)=\frac{D S_{\grave{x}}}{n}
$$

En la evaluación de este parámetro el vial de inyección se sometió a calibración por gravimetría, la desviación estándar de los valores medidos fue de $0.060 \mathrm{~mL}$ con 80 mediciones:

$$
U(v o l)=\frac{0.060}{\sqrt{80}}=0.0067 \mathrm{~mL}
$$

3. La incertidumbre de la concentración del extracto se estimó por la combinación de las incertidumbres en el área y en la interpolación. La desviación estándar de las áreas en las seis repeticiones fue 3953 unidades. En la (Tabla 11) se muestran los resultados.

Tabla 11. Variabilidad en las áreas bajo el pico de forato.

\begin{tabular}{cc}
\hline \multicolumn{2}{c}{ INCERTIDUMBRE EN LA INYECCIÓN } \\
\hline RÉPLICA & ÁREA \\
\hline 1 & 205.95 \\
2 & 214.01 \\
3 & 202.87 \\
4 & 208.76 \\
5 & 204.97 \\
6 & 209.54 \\
\hline Promedio & 207.68 \\
Desviación estándar & 3.95 \\
\%RSD & $\mathbf{1 . 9 0}$ \\
\hline
\end{tabular}

4. Después de esto, el extracto se inyectó en el instrumento para la obtención de los respectivos picos cromatográficos y sus áreas, las cuales se interpolaron en la gráfica de calibrado del instrumento con el fin de determinar la concentración de plaguicida en el extracto. La medición del volumen de in- yección, las pérdidas de analito en el puerto de inyección, la eficiencia de la ionización en la fuente, la pérdida de iones en el cuadrupolo y la respuesta del detector suponen fuentes de incertidumbre en el área medida bajo el pico. Todas estas se cuantificaron de manera global al inyectar seis repeticiones del patrón de referencia con el que se doparon las muestras para evaluar el porcentaje de recuperación, y con la determinanción de la dispersión de las áreas resultantes. La incertidumbre estándar en la inyección está determinada por la ecuación (5).

$$
U(\text { iny })=\frac{\text { Area }}{\sqrt{n}}(5)
$$

Para el forato se determinó la siguiente incertidumbre estándar por inyección de la muestra:

$$
U(\text { iny })=\frac{3953}{\sqrt{6}}=1613.85 \text { unidades de área }
$$

A fin de combinar la incertidumbre de las áreas con la incertidumbre en la interpolación, se multiplicó por su coeficiente de sensibilidad; para calcularlo se derivó la concentración con respecto al área en la ecuación de la línea de calibrado:

$$
[]=\frac{\text { Area }-b}{m}=\frac{\partial[]}{\partial \text { Area }}=\frac{1}{m}=0.00000046
$$

El cálculo de la incertidumbre en la interpolación es un poco más complejo; en primer lugar, se calcula el estadístico $\mathrm{S}_{\mathrm{y} / \mathrm{x}}$ según:

$$
S_{y / x}=\sqrt{\left\{\frac{\sum_{i}\left(y_{i}-y_{i}^{\hat{i}}\right)^{2}}{n-2}\right\}}(7)
$$

Donde $y_{i}$ es el área medida para cada concentración usada en la curva de calibración (en este caso corresponde al promedio de tres repeticiones), ${ }^{\wedge} y_{i}$ es el valor de área corregido o calculado con la ecuación de la gráfica resultante, y n es el número de puntos de calibración (seis en este caso). Obtenido $S_{y / x}$ el error en la interpolación se calcula con:

$$
S_{\left.x_{(}\right)}=\frac{S_{y / x}}{b} \sqrt{\frac{1}{m}+\frac{1}{n}}+\frac{\left(y_{o}-\bar{y}\right)^{2}}{b^{2} \Sigma_{i}\left(x_{i}-x \text { promedio }\right)^{2}}(8)
$$

Donde $b$ es la pendiente de la gráfica de calibrado, $m$ es el número de mediciones del extracto (en este caso se hicieron seis mediciones, pero en el trabajo de rutina las muestras se miden, por lo general, una sola vez), $\mathrm{n}$ nuevamente es el número de puntos de calibrado, $y_{0}$ es el área medida o promedio de áreas medidas para el extracto y es el promedio de las áreas de los patrones ŷ de calibración.

Para el Forato se obtuvieron los siguientes valores:

$$
\begin{gathered}
S_{y / x}=8586.14 \text { unidades de área } \\
U(\text { int })=S_{\left.x_{(}\right)}=0.0028 \mathrm{mg} / \mathrm{L}
\end{gathered}
$$


Finalmente:

$$
U(\text { ext. })=\sqrt{\left[U(\text { Área }) * \frac{\partial_{\text {conc }}}{\partial_{\text {Área }}}\right]^{2}+[U(\text { int })]^{2}}
$$

Para el forato se determinó:

$$
U(\text { ext. })=0.0029 \mathrm{mg} / \mathrm{L}
$$

En cuanto a la interpolación, por ser la gráfica de calibrado un instrumento de medida, tiene una incertidumbre asociada que se cuantificó con cálculos matemáticos basados en los residuales (las diferencias entre los valores de área medidos y los valores de área calculados a partir de la ecuación resultante de la regresión lineal). De manera que el error en la concentración del extracto se cuantificó al combinar la incertidumbre en el área con el error en la interpolación (24.26).

\section{Coeficientes de sensibilidad}

El coeficiente de sensibilidad describe qué tan sensible es el mensurando con respecto a variaciones de la magnitud de entrada correspondiente. Se calcula mediante la derivada parcial de la función que relaciona las variables de entrada con respecto a la correspondiente variable de entrada [24].

Coeficiente de sensibilidad de la pesada

$$
\frac{\partial_{C_{m}}}{\partial_{m_{m}}}=100 \frac{C_{\text {ext }} * V_{\text {ext }}}{\% R * m_{m^{2}}}(10)
$$

Coeficiente de sensibilidad de la recuperación

$$
\frac{\partial C_{m}}{\partial \% R}=100 \frac{C_{\text {ext }} * V_{\text {ext }}}{\% R^{2} * m_{m}}(11)
$$

Coeficiente de sensibilidad de volumen de extracto

$$
\frac{\partial C_{m}}{\partial V_{\text {ext }}}=100 \frac{C_{\text {ext }}}{\% R * m_{m}}(12)
$$

Coeficente de sensibilidad de concentración del extracto

$$
\frac{\partial C_{m}}{\partial C_{\text {ext }}}=100 \frac{V_{\text {ext }}}{\% R * m_{m}} \text { (13) }
$$

A fin de obtener la incertidumbre combinada, que a la vez es la incertidumbre estándar de la variable de salida (concentración del plaguicida en la muestra de café verde), se estimó el peso que cada una de las incertidumbres estándar calculadas anteriormente tienen sobre el resultado final. Esto se consiguió al calcular el producto de cada incertidumbre estándar por el coeficiente de sensibilidad, entonces, la incertidumbre combinada se obtuvo mediante:

$\boldsymbol{U}\left(\boldsymbol{C}_{\boldsymbol{m}}\right)=$
$\sqrt{\left(\boldsymbol{u}(\text { ext. }) * \frac{\partial C_{m}}{\partial C_{\text {ext }}}\right)^{2}+\left(\boldsymbol{u}(\boldsymbol{v o l}) * \frac{\partial C_{m}}{\partial V_{\text {ext }}}\right)^{2}+\left(\boldsymbol{u}(\boldsymbol{m}) * \frac{\partial C_{m}}{\partial m_{m}}\right)^{2}+\left(\boldsymbol{u}(\% \boldsymbol{R}) * \frac{\partial C_{m}}{\partial \% \boldsymbol{R}}\right)^{2}}$

Se utilizó el factor de cobertura k = 2 para calcular la incertidumbre expandida con un nivel de confianza del $95.45 \%$.

$$
U\left(C_{m}\right)=0.00065 * 2=0.0013 \mathrm{mg} / \mathrm{Kg}
$$

El resultado final para la concentración de forato en la muestra se calculó con la ecuación (1), y el resultado final fue:

$$
C_{m}=0.0098 \pm 0.0013 \mathrm{mg} / \mathrm{Kg}
$$

\section{Análisis de muestras}

En la (Tabla 12) se relacionan los resultados para todos los plaguicidas evaluados con sus respectivas incertidumbres. Allí se puede observar que en la última columna de la tabla se muestran las incertidumbres relativas para el forato, la concentración final es de $0.0098 \mathrm{mg}$ por Kg de café verde, con una incertidumbre de $0.0013 \mathrm{mg} / \mathrm{Kg}$, lo que corresponde al $13,4 \%$ del valor de concentración. A fin de evaluar la exactitud final del método se calculó el porcentaje de error en la concentración; los resultados se muestran en la (Tabla 13).

Finalmente, se tiene que los porcentajes de error en la concentración medida estuvieron por debajo del $6 \%$, con incertidumbres inferiores al $13.5 \%$, a un grado de confiabilidad del 95\%. En consecuencia, la metodología propuesta en este trabajo es válida para cuantificar los plaguicidas forato, terbufós, disulfotón, clorpirifós, triadimefón, triadimenol y propiconazol en café verde tipo exportación.

Tabla 12. Resultados de la cuantificación de plaguicidas en café verde.

\begin{tabular}{cccc}
\hline PESTICIDA & CONCENTRACIÓN & $\mathrm{U}^{*}$ & U RELATIVA \\
\hline Forato & 0.0098 & 0.0013 & 13.4 \\
Terbufós & 0.026 & 0.0020 & 7.7 \\
Disulfotón & 0.051 & 0.0041 & 8.0 \\
Clorpirifós & 0.025 & 0.0030 & 12.0 \\
Triadimefón & 0.026 & 0.0020 & 7.6 \\
Triadimenol & 0.047 & 0.0059 & 12.5 \\
\hline Propiconazol I \& II & 0.048 & 0.0041 & 8.6 \\
\hline
\end{tabular}

*U: incertidumbre en cuantificación de plaguicidas en café verde.

Tabla 13. Error en el cálculo de concentración de plaguicidas en café verde.

\begin{tabular}{cccc}
\hline PESTICIDA & $\begin{array}{c}\text { CONCENTRACIÓN } \\
\text { ESPERADA }\end{array}$ & $\begin{array}{c}\text { CONCENTRACIÓN } \\
\text { CALCULADA }\end{array}$ & \% ERROR \\
\hline Forato & 0.010 & 0.0098 & 2.0 \\
Terbufós & 0.025 & 0.026 & 2.4 \\
Disulfotón & 0.050 & 0.051 & 2.2 \\
Clorpirifós & 0.025 & 0.025 & 1.2 \\
Triadimefón & 0.025 & 0.026 & 4.0 \\
Triadimenol & 0.050 & 0.047 & 5.8 \\
Propiconazol I \& II & 0.050 & 0.048 & 3.8 \\
& & &
\end{tabular}




\section{CONCLUSIONES}

Los resultados de la estandarización de la técnica multirresiduo empleada para determinar plaguicidas organoclorados y triazoles en café verde tipo exportación indican que la exactitud y la precisión (\%RSD), en todos los casos, fueron mayores al 94\% para cinco niveles de concentración. Las correlaciones de las curvas de calibración oscilaron entre 0.996 y 0.999 , y para rangos lineales de trabajo entre 0.005 y $0.800 \mathrm{ppm}$. Los límites de detección fueron en todos los casos inferiores a $0.025 \mathrm{ppm}$, de manera que cumplieron con las necesidades legislativas. Los porcentajes de recuperación oscilaron entre el $71.9 \%$ y $93.9 \%$, con dispersiones inferiores al $9.13 \%$. El efecto de matriz influyó sobre los plaguicidas terbufós, clorpirifós y triadimefón, en los que sus límites de detección se duplicaron. Para el cálculo global de la incertidumbre se tuvieron en cuenta las fuentes más significativas del proceso y el resultado final fue, para todos, inferior al $13.5 \%$ de incertidumbre, con lo cual se cumplieron los criterios de aceptación en análisis de residuos de plaguicidas.

\section{AGRADECIMIENTOS}

Los autores presentan sus agradecimientos a los productores pertenecientes a la Federación Nacional de Cafeteros que facilitaron la matriz de estudio, y a la Universidad Tecnológica de Pereira por su apoyo con los recursos necesarios para la realización del proyecto.

\section{REFERENCIAS}

- Aguirre, L., Pérez, J. A. y Pujol, M. (2001). Validación de métodos analíticos. Barcelona: Asociación Española de Farmacéuticos de la Industria.

- Dallos, D. y Guerrero, J. (2005). Desarrollo y validación de una metodología para la determinación de plaguicidas en café verde por cromatografía de gases. Revista Colombiana de Química, 34(2), 175-188. doi: https://doi. org/10.15446/rev.colomb.quim.

- Dinero. (2015). ¿Colombia exporta café a los mayores consumidores del mundo? Recuperado de http://www.dinero.com/economia/articulo/colombia-exporta-cafe-mayores-consumidores-del-mundo/210780.

- Ellison, S. L. R., Williams, A. y Eurachem Working Group on Uncertainty in Chemical Measurement. (2012). Quantifying uncertainty in analytical measurement. Eurachem.

- Federación Nacional de Cafeteros. (2017). Producción de café de Colombia aumenta $12 \%$ en enero. Recuperado de https://www.federaciondecafeteros.org/ clientes/es/sala_de_prensa/detalle/produccion_de_cafe_de_colombia_aumenta_12_en_enero/.

- Guo, Q., Lv, X., Tan, L. y Yu, B-Y. (2009). Simultaneous Determination of 26 Pesticide Residues in 5 Chinese Medicinal Materials Using Solid-phase Extraction and GC-ECD Method. Chinese Journal of Natural Medicines, 7(3), 210216. doi: https://doi.org/10.1016/S1875-5364(09)60054-6.

- Instituto Colombiano Agropecuario. Subgerencia Protección y Regulación Agrícola. (2017). Restricciones, prohibiciones y suspensión de registros de plaguicidas de uso agrícola en Colombia.

- Japan Mistry of Health, Labour and Welfare. (2006). Analytical Methods for Residual Compositional Subtances of Agricultural Chemicals, Feeds Additives, and Veterinary Drugs in Food Deparment of Food Safety. Recuperado de http:// ww.mhlw.go.jp/english/topics/foodsafety/positivelist060228/dl/060526-1a.pdf.
- Juraske, R., Mutel, C. L., Stoessel, F. y Hellweg, S. (2009). Life Cycle Human Toxicity Assessment of Pesticides: Comparing Fruit and Vegetable Diets in Switzerland and the United States. Chemosphere, 77(7), 939-945. doi: https:// doi.org/10.1016/j.chemosphere.2009.08.006.

- Landau-Ossondo, M., Rabia, N., Jos-Pelage, J., Marquet, L. M., Isidore, Y., Saint-Aimé, C.... y Belpomme, D. (2009). Why Pesticides Could be a Common Cause of Prostate and Breast Cancers in the French Caribbean Island, Martinique. An Overview on Key Mechanisms of Pesticide-Induced Cancer. Biomedicine \& Pharmacotherapy, 63(6), 383-395. doi: https://doi.org/10.1016/j. biopha.2009.04.043.

- Muñoz, L. (2012). Café: principal dinamizador de la economía rural. https:// www.portafolio.co/negocios/empresas/cafe-principal-dinamizador-economiarural-105724

- Órgano Nacional de Acreditación de la República de Cuba. (2016). Política de Incertidumbre de las Medición Revo4, ONARC POL 3 §.

- Pizzutti, I. R. y De Kok, A., Dickow Cardoso, C., Reichert, B., de Kroon, M., Wind, W. ... y Caiel da Silva, R. (2012). A Multi-Residue Method for Pesticides Analysis in Green Coffee Beans Using Gas Chromatography-Negative Chemical Ionization Mass Spectrometry in Selective Ion Monitoring Mode. Journal of Chromatography A, 1251, 16-26. doi: https://doi.org/10.1016/j.chroma.2012.06.041.

- Resolución Número 2906 de 2007 [Ministerio de Agricultura y Desarrollo Rural y Ministerio de la Protección Social]. Por la cual se establecen los Límites Máximos de Residuos de Plaguicidas-LMR en alimentos para consumo humano y en piensos o forrajes. Agosto 22 de 2007. DO 46735.

- Schmid, W. A., Lazos, R. J. y Revisión, M. (2004). Guía para estimar la incertidumbre de la medición. Centro Nacional de Metrología.

- World Health Organization y Food and Agriculture Organization of United Nations. (2017). Codex Alimentarius: Pesticide MRLS.

- Yang, X., Zhang, H., Liu, Y., Wang, J., Zhang, Y. C., Dong, A. J. ... y Cui, J. (2011). Multiresidue Method for Determination of 88 Pesticides in Berry Fruits Using Solid-Phase Extraction and Gas Chromatography-Mass Spectrometry: Determination of 88 Pesticides in Berries Using SPE and GC-MS. Food Chemistry, 127(2), 855-865. doi: https://doi.org/10.1016/j.foodchem.2011.01.02. 